\title{
TYPE II BURSTS AT HECTOMETRIC AND KILOMETRIC WAVELENGTHS FROM INTERPLANETARY SHOCKS*
}

\author{
H. H. MALITSON, J. FAINBERG, and R. G. STONE \\ NASA-Goddard Space Flight Center, Greenbelt, Md. 20771, U.S.A.
}

The first type II radio burst to be observed at frequencies below the ionospheric cutoff was recorded by the Goddard radio astronomy experiment on the Interplanetary Monitoring Platform, IMP-6. It occurred on June 30, 1971; a tracing made from the intensity contour plot of the radio data is shown in Figure 1. On the original, intensity contours were plotted in $\mathrm{dB}$ above background as a function of frequency and time. The figure shows 32 discrete frequencies from $4.9 \mathrm{MHz}$ to $30 \mathrm{kHz}$ received by the IMP-6 experiment. The time scale covers a period of almost five hours. During this time, five individual type III bursts and one group of type III's occurred; they are shown by their outlines to be drifting rapidly from high to low frequencies. The relatively narrow-band, slowly-drifting radiation with clearly defined fundamental and second harmonic is the type II. The average drift rate, represented by the straight lines drawn through the burst, is approximately $2.5 \mathrm{kHz} \mathrm{min}^{-1}$ in this frequency range, and is roughly 100 times slower than that of the type III bursts. The ovals defining the type II emission were traced from the record and show modulation of the signal caused by the spin of the satellite and the resultant sweeping of the antenna pattern of the dipole across the source direction. On the original record, the outlines of the type III's are also filled with a pattern of modulation ovals that were omitted from the figure for clarity. The shaded portion of the record at the lower frequencies represents magnetospheric noise.

Figure 2 shows data from the IMP-6 experiment for August 7 and 8, 1972. The 3B flare at about 1500 UT on August 7 is represented by a dot at the upper left. Its associated shock front produced the sudden-commencement geomagnetic storm beginning at 2354 UT on August 8, the dot at the lower right corner of the figure. The straight line drawn between them represents the average velocity of the shock front over the $1 \mathrm{AU}$ distance, about $1270 \mathrm{~km} \mathrm{~s}^{-1}$. The horizontal lines in areas B, C, and D represent the times during which type II radiation at various frequencies was observed. This radiation is not observed all the time, but is sporadic - a characteristic of many type II bursts observed from the ground. Apparently, special physical conditions are necessary to produce type II emission which are not always present as the shock front proceeds outward.

\footnotetext{
* Summary of the following two papers:

'Observation of a Type II Solar Radio Burst to $37 R_{\odot}$ ', H. H. Malitson, J. Fainberg, and R. G. Stone, Astrophys. Letters 14, 111-114, 1973.

'A Density Scale for the Interplanetary Medium from Observations of a Type II Solar Radio Burst out to 1 Astronomical Unit', H. H. Malitson, J. Fainberg, and R. G. Stone, Astrophys. J. 183, L35-L38, 1973.
} 

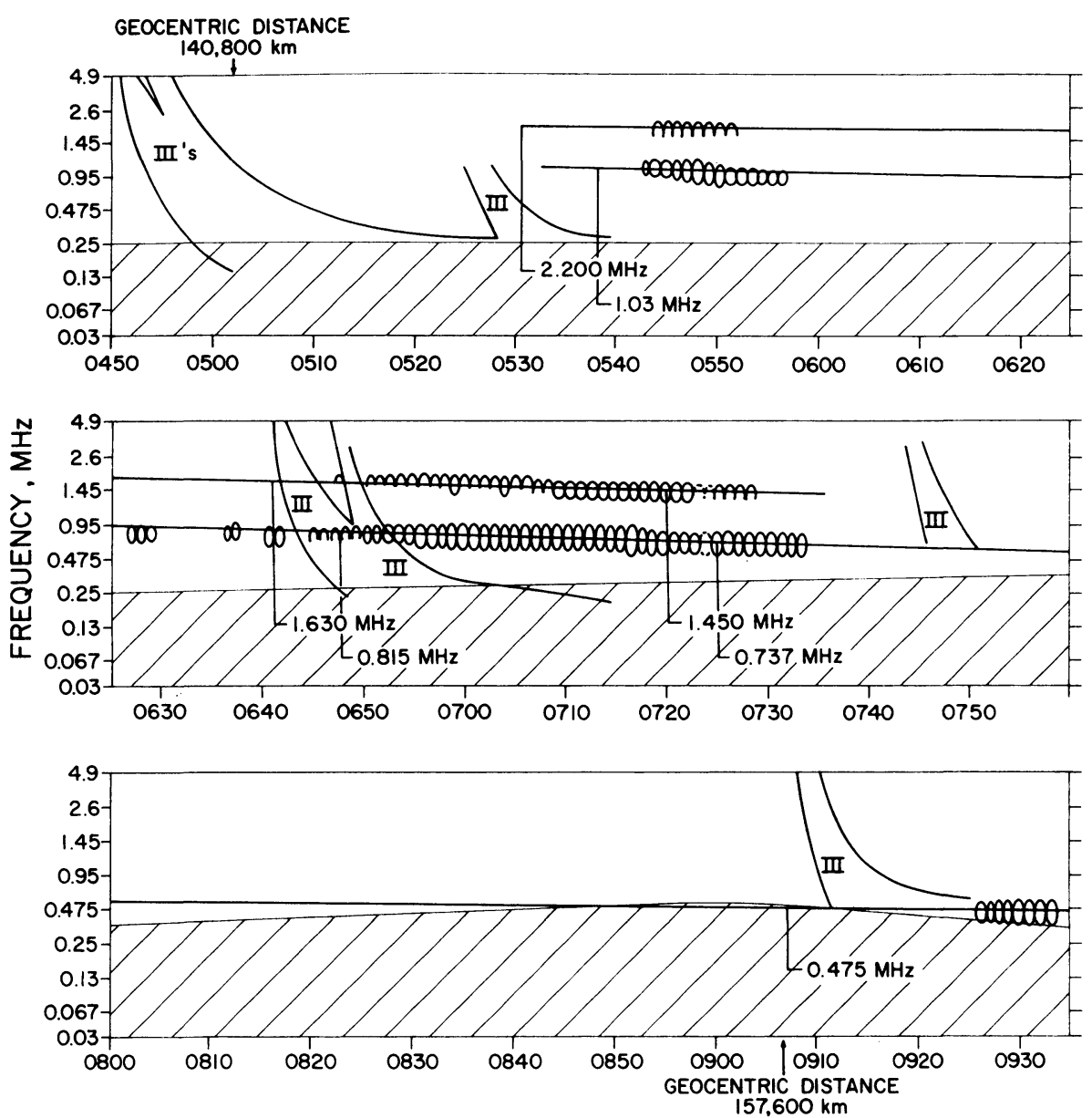

UT,JUNE 30,1971

Fig. 1. [Courtesy of Astrophys. Letters, Gordon and Breach.]

Type II emission is commonly observed in all its wavelength ranges, both at the plasma frequency characteristic of its point of origin in the corona and at the second harmonic of that frequency. This is also true of the present data, at least in regions B and $\mathrm{C}$ where the burst is quite strong and well defined. Only the observations of the fundamental have been used in the figure, since those at the harmonic would tend to overlap the others.

In placing the lines for the different frequencies at certain points along the vertical distance scale, the coronal emission levels derived by Fainberg and Stone (1971) were used. These investigators developed a method, using a hectometric storm of type III bursts observed with the satellite RAE-1 over a half solar rotation, to derive a density scale in the range $10-40 R_{\odot}$. In addition, the assumption was made that the observed type III's in the hectometric storm were all at the plasma frequency. The 


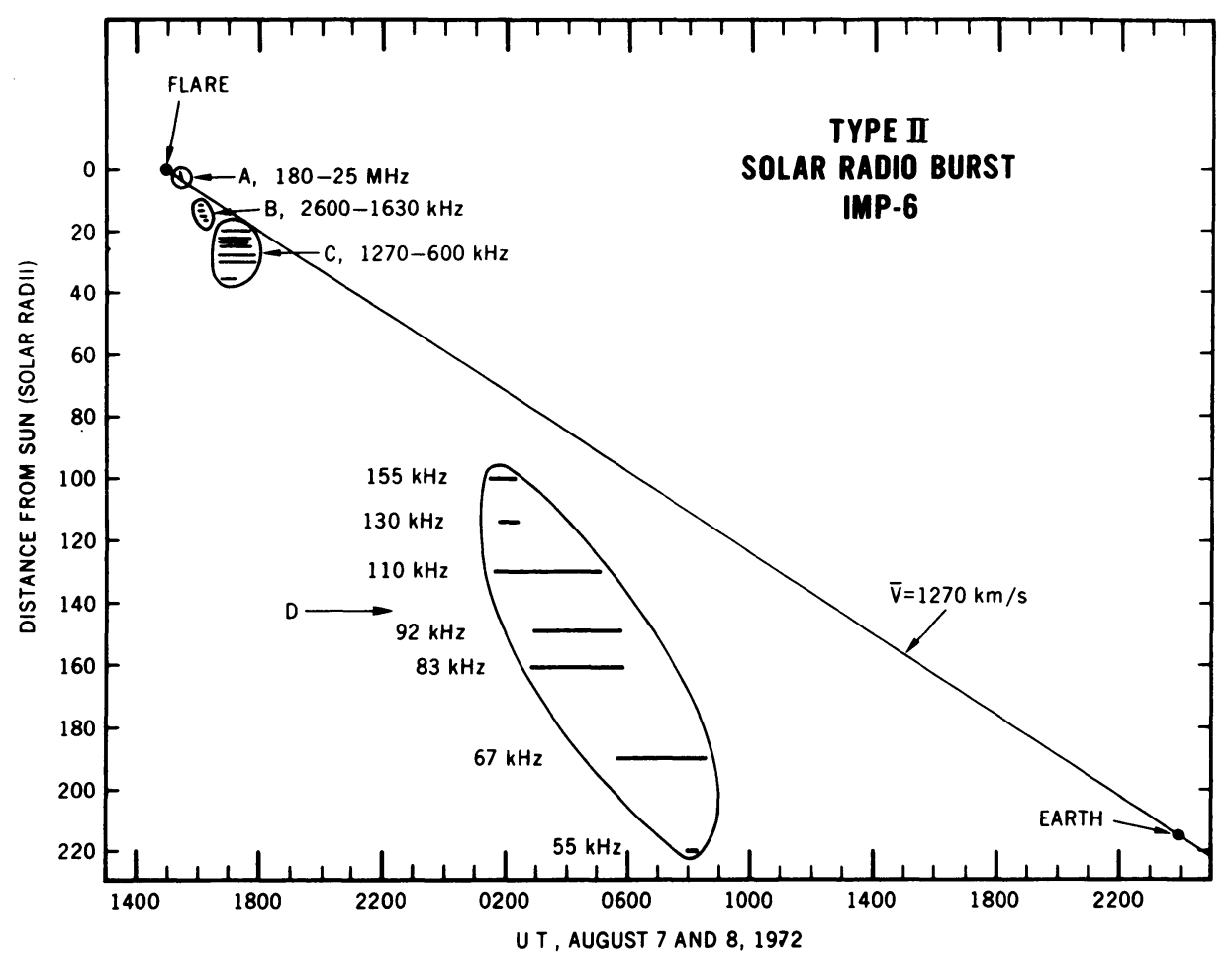

Fig. 2. [Courtesy of Astrophys. J., Univ. of Chicago Press.]

lines having been arranged vertically, it is easy to see that the corresponding velocity out from the Sun is about twice the average velocity of $1270 \mathrm{~km} \mathrm{~s}^{-1}$, and would result in the premature arrival of the shock front at the Earth.

For Figure 3, the same analysis was used except this time the assumption was made that type III bursts at hectometric and kilometric wavelengths are seen at twice the plasma frequency. In this case, the IMP-6 data fall in regions $\mathbf{B}^{\prime}, \mathbf{C}^{\prime}, \mathbf{D}^{\prime}$, and $\mathrm{E}^{\prime}$. Note in particular $\mathrm{E}^{\prime}$, the observation at $30 \mathrm{kHz}$, just before the sudden commencement of the geomagnetic storm. The measured position of the burst at this frequency was $20^{\circ}$ east of the Sun. The $30-\mathrm{kHz}$ data were omitted from Figure 2, where they would have fallen considerably below the bottom edge of the scale, at about $350 R_{\odot}$. At this distance, the burst probably could not have been observed through the intervening plasma.

In Figure 3, the fit of the velocity line to the IMP-6 data is much better than in Figure 2. The agreement is remarkably good, considering the inhomogeneities in electron density along the path, the overall variation in density with time, the possible variations in velocity of the shock front, and the sporadic nature of the type II emission. This agreement, we feel, is strong evidence for the adopted density scale and strongly implies that hectometric and kilometric type III bursts are observed at twice the source plasma frequency. 
We are in the process of studying more type II bursts, and expect the data to provide important contributions to the study of the interplanetary medium in two ways:

(1) As the type II bursts travel outward, the changes in intensity and variations in velocity should provide valuable information on the propagation of shock waves through the medium. With the remote sensing techniques of radio astronomy, the

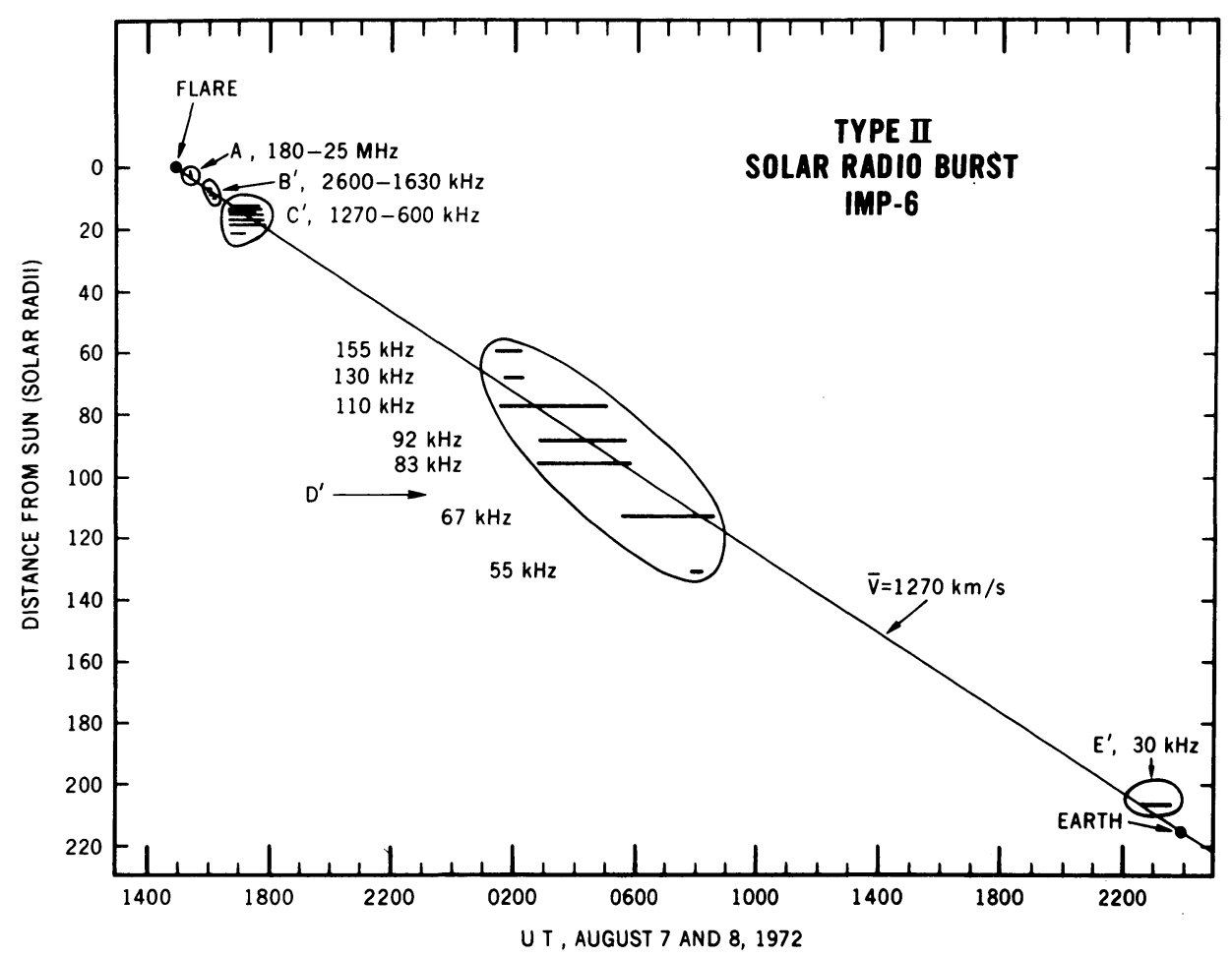

Fig. 3. [Courtesy of Astrophys. J., Univ. of Chicago Press.]

investigation of shock waves is not limited to those traveling only in the ecliptic plane near $1 \mathrm{AU}$.

(2) With type II bursts having well-defined first and second harmonic components as probes, the electron-density scale of the interplanetary medium can be determined unambiguously from regions close to the Sun out to regions close to $1 \mathrm{AU}$.

\section{Reference}

Fainberg, J. and Stone, R. G.: 1971, Solar Phys. 17, 392.

\section{DISCUSSION}

Maxwell: Does the Earth noise arise in the magnetosphere, and is it wiped out when the shock reaches the Earth? 
Malitson: Yes, it is magnetosphere noise but it is not wiped out; indeed, if anything, it seems to be enhanced.

Fainberg: This noise is always variable and we cannot clearly tell whether it is stronger or weaker after the shock.

Zirin: Is there any comparison between the trajectory for this shock and those of August 2 and 4 ?

Malitson: The corresponding records are being searched, but isolating a type II from other types of strong emission is difficult.

Smith: I see that type II burst observations combined with type III observations can provide a density model. What does a type II look like at low frequencies? Is it better or worse than a type III burst for making a density model?

Malitson: We only see the centroid of the burst so we can tell nothing about its shape or size.

Kai: I wonder why type II bursts are so rarely observed in the interplanetary medium, since the close relationship between type II bursts and the Earth's magnetic storms is well established. Is this due simply to technical problems?

Malitson: It may be that special physical conditions are necessary for a shock front to produce radio emission - e.g., the crossing of the front by a type III. Also, type II's are very sporadic and broken and it is hard to find even the strongest bursts in the records.

Dryer: In partial answer to Dr Kai's comment about other measurements of interplanetary type II's and correlation with geomagnetic storms, I might add that the first such measurement (if I recall correctly) was made by Slysh. There was a geomagnetic storm correlated with his observations on the Soviet space probe.

Malitson: That measurement was made at only two frequencies and there is some doubt that it really was a type II.

Dryer: You may be right; I do not recall if there were two or more.

Sakurai: When we compare SSC's with interplanetary shock waves as observed by satellites, we find that some SSC's are produced by interplanetary shocks, whereas the others are produced by discontinuous, or sharp, variations of interplanetary magnetic fields associated with enhanced solar winds. Therefore, there is no one-to-one correspondence between an SSC and an interplanetary shock wave. This means that there is not much chance for type II bursts to be excited in interplanetary space.

McLean : Was there any position difference between the fundamental and second harmonic component?

Malitson: There was none found within our accuracy of $1^{\circ}$ to $2^{\circ}$.

Smerd: Ms Malitson noted that the interplanetary electron densities derived from interplanetary observations of type III and type II bursts could be made to agree on the assumption that the type III radiation was at the second harmonic. The same agreement can be achieved if we assume that the type III radiation is at the fundamental plasma frequency, $f_{p}$, while the fundamental of the type II burst was radiated at the enhanced plasma frequency, $f_{p}($ shock $) \sim 2 f_{p}$, behind a strong type II shock. 